\title{
A Most Energetic Type Ic Supernova: SN 2003L
}

\author{
Alicia M. Soderberg \\ Palomar Observatory, 105-24, California Institute of Technology, Pasadena, CA \\ 91125, USA; \\ ams@astro.caltech.edu
}

Summary. We present extensive radio observations of SN2003L, the most luminous and energetic Type Ic radio supernova with the exception of SN 1998bw. Using radio data, we are able to constrain the physical parameters of the supernova, including the velocity and energy of the fastest ejecta, the temporal evolution of the magnetic field, and the density profile of the surrounding medium. We highlight the extraordinary properties of the radio emission with respect to the supernova's normal characteristics within optical bands. We find that although the explosion does not show evidence for a significant amount of relativistic ejecta, it produces a radio luminosity which is comparable to that seen in the unusual SN1998bw. Using SN2003L as an example, we comment briefly on the broad diversity of type Ic properties and the associated implications for progenitor models.

\section{Introduction}

Despite active campaigns to study radio emission from type $\mathrm{Ib} / \mathrm{c}$ supernovae, only a small number of events have been successfully detected. Among the class of radio bright supernovae is $\mathrm{SN} 1998 \mathrm{bw}$, an unusually bright type Ic discovered within the error box of the nearby gamma-ray burst GRB 980425 . Reaching a peak radio luminosity $\sim 100$ times higher than all other radio bright type Ib/c supernovae (SNe), it has been proposed that SN1998bw was powered by a central engine, similar to the popular model for gamma-ray bursts (GRBs) [3].

In this paper we present observations of the first radio bright type $\mathrm{Ib} / \mathrm{c}$ supernova with energetics comparable to those shown in SN1998bw. SN2003L was optically discovered on 2003 Jan 12.15 UT [2] and spectroscopically identified on 2003 Jan 25.0 UT [5, 8]. The supernova was seen to bear strong resemblance to the typical type Ic SN1994I at maximum light, showing low average expansion velocities of $5900 \mathrm{~km} / \mathrm{s}$ as derived from the Si II line. The optical lightcurve peaks at $m_{V} \approx 16$ which places SN2003L among the brightest optical type Ic's observed to date. 

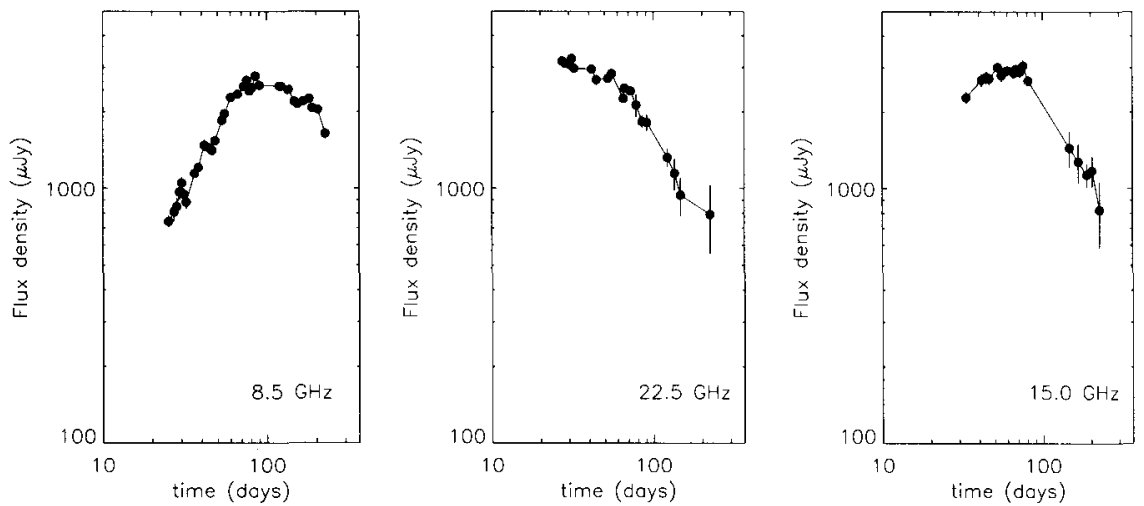

Fig. 1. Radio lightcurves for type Ic SN 2003L at 8.5, 15., and $22.5 \mathrm{GHz}$ for the time period 2003 Jan 26 - 2003 Aug 15 UT.

\section{Observations with the VLA}

On 2003 Jan 26.23 UT we detected a radio transient coincident with the optical position of SN2003L. We subsequently initiated an intense radio monitoring campaign at the Very Large Array (VLA) to trace the evolution of the radio emission from the supernova. Data were taken in standard continuum observing mode with a bandwidth of $2 \times 50 \mathrm{MHz}$ centered on frequencies 8.5 , 15.0 and $22.5 \mathrm{GHz}$. Flux density measurements were derived using calibrator $3 \mathrm{C} 286$ and phase referenced against calibrators J1118+125, J1120+134, and J1103+119. Data were reduced using standard packages within the Astronomical Image Processing System (AIPS). At $8.5 \mathrm{GHz}$ (our most densely sampled light-curve) typical flux uncertainties were $\sim 60 \mu \mathrm{Jy}$ for an average integration time of 10 minutes. The results of our radio monitoring campaign of SN2003L are summarized as Fig. 1. These observations demonstrate a broad spectrum, similar to that observed for SN 1998bw.

\section{Robust Constraints}

Here we discuss the constraints imposed by the radio observations for SN2003L. As a preliminary constraint on the total energy of the source, we estimate the brightness temperature $\left(T_{b}\right)$ of the supernova and compare it with the robust constraints imposed by equipartition arguments and the inverse Compton catastrophe (ICC). From [6], the brightness temperature is defined as a function of the observed flux density, the peak frequency, and the angular size of the source. As an initial estimate for the physical size of the SN ejecta, we first assume the optical expansion velocity of $5900 \mathrm{~km} \mathrm{~s}^{-1}$ [8] can be used as an average speed to describe the motion of the radio bright 
ejecta. Using an approximate explosion date of 2003 Jan 1 UT based on optical light-curves [7], we estimate the shock radius to be $r \approx 4.3 \times 10^{15} \mathrm{~cm}$ at $t \approx 85$ days when the observed flux density peaked at $8.5 \mathrm{GHz}$. Using the observed peak flux of $F_{8.5 \mathrm{GHz}} \approx 2.8 \mathrm{mJy}$ and adopting a source distance of $91.7 \mathrm{Mpc}\left(\Omega_{M}=0.27, \Omega_{\Lambda}=0.73, H_{0}=71 \mathrm{~km} \mathrm{~s}^{-1} \mathrm{Mpc}^{-1}\right)$, we find a brightness temperature of $T_{b} \approx 1.8 \times 10^{12} \mathrm{~K}$ which is dangerously near the ICC limit of $T_{b}<10^{12} \mathrm{~K}$. This suggests that the radio ejecta expanded with a velocity significantly higher than that observed at optical frequencies. In fact, an ejecta velocity of $\sim 16,000 \mathrm{~km} \mathrm{~s}^{-1}(\sim 3$ times larger than that derived from optical spectroscopy) would be necessary to avoid violating the ICC constraint.

Assuming equipartition of energy places a further constraint on the brightness temperature limit and reduces it to $T_{b}<5 \times 10^{10} \mathrm{~K}$. Using the equipartition arguments of [6] and [3], we derive the minimum energy for the radio supernova. Assuming that the observed radio flux is produced by synchrotron emission, the total energy of the source $(U)$ can be expressed as the sum of the energy in relativistic electrons $\left(U_{\mathrm{e}}\right)$ and the energy in the magnetic field $\left(U_{\mathrm{B}}\right)$. At equipartition, the fraction of total energy in electrons equals the fraction of total energy in magnetic fields $\left(\epsilon_{e}=\epsilon_{B}=1\right)$ and the total energy is minimized at $U_{\text {eq }}[6]$. This occurs when the emitting source reaches an equipartition radius denoted by the angular size, $\theta_{\text {eq }}$. The minimum energy of the source can be thus be parameterized in terms of the synchrotron peak flux and the equipartition size.

Using our most densely sampled light-curve, we fit for the peak flux $\left(S_{p}\right)$ over the observed $\sim 200$ day evolution and find $S_{p} \approx 2.8 \mathrm{mJy}$ at $\nu_{p}=8.5 \mathrm{GHz}$ on 2003 Mar 27 UT $(\approx 85$ days since explosion). For this epoch we estimate an angular size $\theta_{\mathrm{eq}} \approx 19 \mu \mathrm{as}$ (with $\beta \approx-1.0$ ) which implies an average shock velocity of $v \approx 0.1 c$ and an equipartition brightness temperature of $T_{b_{\text {eq }}} \approx$ $5.0 \times 10^{10} \mathrm{~K}$. By setting $U=U_{\mathrm{eq}}$ we find the energy is minimized at the equipartition value of $U_{\text {eq }} \approx 4.3 \times 10^{47} \mathrm{erg}$ with an associated magnetic field strength of $B_{p_{\mathrm{ec}}} \approx 0.6 \mathrm{G}$. As shown by [6], synchrotron emission systems which diverge from equipartition necessitate a huge increase in total energy. Consequently, it is possible that the total energy contained within the fast moving ejecta of SN2003L is in fact much larger than $4.3 \times 10^{47}$ ergs.

These preliminary constraints allow us to make two robust conclusions:

1. The velocity of the radio bright $\mathrm{SN}$ ejecta must be at least $16,000 \mathrm{~km} \mathrm{~s}^{-1}$ to avoid violating the inverse Compton catastrophe limit and $\sim 30,000$ $\mathrm{km} \mathrm{s}^{-1}$ assuming equipartition.

2. The energy of the supernova must be $>4.3 \times 10^{47}$ erg and could be significantly larger depending on the proximity of the system to equipartition.

These conclusions imply that there was a considerable amount of energy released at high velocities in the type Ic supernova explosion of SN2003L. In fact, these equipartition constraints alone demand that SN2003L is among the 


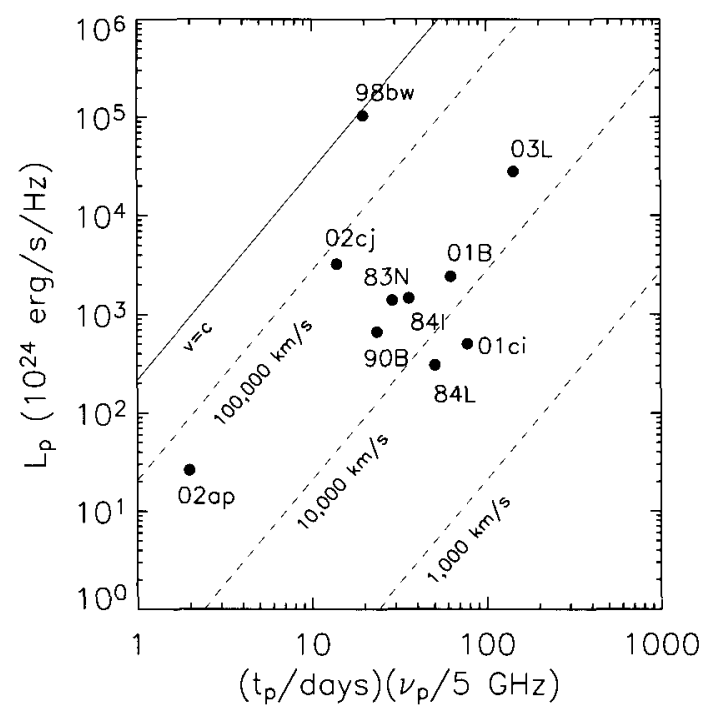

Fig. 2. The diversity of peak luminosities and observed time of the peak is shown above for all radio bright type $\mathrm{Ib} / \mathrm{c}$ supernova. By assuming equipartition, the expansion velocity for each event can be estimated (dashed lines). Note the radio ejecta of SN2003L is relatively slow and luminous.

most energetic type $\mathrm{Ib} / \mathrm{c}$ supernovae observed to date, second only to the unusual event of SN1998bw/GRB980425. Fig. 2 is a compilation of all the radio bright type $\mathrm{Ib} / \mathrm{c}$ supernovae observed to date. By comparing the peak radio luminosity to the observed time of peak flux, the diversity in equipartition derived expansion velocities can be examined. Note that although SN2003L peaks later, it is among the brightest radio supernovae.

\section{Implications and Physical Parameters}

Assuming equipartition values of $\epsilon_{B}=\epsilon_{e}=1$ and adopting synchrotron self-absorption as the dominant absorption process, we determine the temporal evolution of the total energy and radius of the ejecta for SN2003L. For observations spanning $t=25-200$ days, the total energy increases from $E \approx 3.0 \times 10^{47}$ to $1.1 \times 10^{48} \mathrm{ergs}$. Over the same period, the shock radius scales as $r \propto t^{0.67}$ from $r \approx 1.45 \times 10^{16}$ to $5.0 \times 10^{16} \mathrm{~cm}$. Assuming an explosion date of $2003 \mathrm{Jan} 1 \mathrm{UT}$, the average velocity decreases from $v \approx 0.22 c$ to $0.10 \mathrm{c}$. In comparison to SN1998bw where it was observed that $v \approx c$, it is clear that SN 2003L does not have a significant amount of material moving at relativistic speeds. However, the total energy within the radio bright ejecta remains quite high at $\sim 10 \%$ that of SN1998bw (on similar timescales) and $10-100$ times greater than other radio supernovae. 
Using the values derived for the total energy and radius, we predict the magnetic field decreased from $B \approx 0.75 \mathrm{G}$ to $0.23 \mathrm{G}$ over the period $t=25-$ 200 days. We find an temporal evolution of $B \propto t^{-0.63}$, such that $B \propto r^{-0.93}$. Extrapolating, we find $B(t) \approx 10 \mathrm{G}$ at $r \approx 10^{15} \mathrm{~cm}$. For comparison, type IIb SN1993J exhibited a similar evolution with $B \approx 60 \mathrm{G}$ at $r \approx 10^{15} \mathrm{~cm}$ and a radial scaling of $B(r) \propto r^{-1}$.

Environmental properties can also be predicted based on our radio observations. From 25 days to 200 days, we find that the electron density, $n_{e}(t)$, drops by a factor of $\sim 10$ from $n_{e} \approx 340 \mathrm{~cm}^{-3}$ to $36 \mathrm{~cm}^{-3}$. Expressed in terms of a radial dependence, $n_{e} \propto r^{-1.8}$; similar to the density profile expected from a massive stellar wind, $n_{e} \propto r^{-2}$. This was also seen in the case of SN1998bw/GRB980425 [4], although solid evidence for a wind environment has yet to be detected for the majority of observed gamma-ray bursts.

Directly coupled to the electron number density is the mass loss rate, $\dot{M}$ of the progenitor star. For an assumed stellar wind velocity of $w=10^{3} \mathrm{~km} \mathrm{~s}^{-1}$, we find a roughly constant mass loss rate of $\dot{M}(t) \approx 5 \times 10^{-6} \mathrm{M}_{\odot} \mathrm{yr}^{-1}$. This is $\sim 10$ percent larger than values derived for SN1998bw and SN2002ap, of $\dot{M}(t) \approx 2.5 \times 10^{-7}$ and $5 \times 10^{-7} \mathrm{M}_{\odot} \mathrm{yr}^{-1}$, respectively $[1,4]$. It should be noted that these rates are consistently smaller than the predicted mass loss rate for Wolf-Rayet stars $\left(\dot{M}(t) \approx 10^{-4}-10^{-5} \mathrm{M}_{\odot} \mathrm{yr}^{-1}\right)$, which are thought to be the progenitors of type Ic supernovae.

\section{Discussion}

Although the constraints provided by equipartition arguments are robust, they are also preliminary. The extensive radio data set for SN2003L warrants a full modeling effort to accommodate the effects of multiple absorption processes including synchrotron self-absorption and free-free absorption. Results from our radio modeling study of SN2003L will be presented along with extensive broadband data for this event [7]. We will show that the highly energetic supernovae SN2003L is becoming one of the best studied radio supernovae to date, thereby offering new insights on the diversity of cosmic explosions.

\section{References}

1. E. Berger, S.R. Kulkarni, R.A. Chevalier: Astrophys. J. Lett. 577, L5 (2002)

2. T. Boles: IAUC 8048 (2003)

3. S.R. Kulkarni et al. : Nature 295, 663 (1998)

4. Z. Li, R.A. Chevalier: Astrophys. J. 526, 716 (1999)

5. T. Matheson, P. Challis, R.P. Kirshner, P.M. Garnavich: GCN 1846 (2003)

6. A.C. Readhead: Astrophys. J. 426, 51 (1994)

7. A.M. Soderberg: in preparation

8. S. Valenti et al. : IAUC 8057 (2003) 